\title{
ON THE TOPOLOGICAL REDUCTION OF FINITE VON NEUMANN ALGEBRAS
}

\author{
Dedicated to Professor Masanori Fukamiya on his 60th birthday \\ Hideo TaKemoto and Jun Tomiyama
}

(Received April 28, 1972; Revised January 22, 1973)

In the present paper, we shall investigate an extension of the Gelfand representation of commutative $C^{*}$-algebras to finite von Neumann algebras from the point of view of continuous reduction theory. Comparing with the successful treatment of this method in commutative cases, the reduction theory of non-commutative von Neumann algebras seems to have been, somewhat, depending upon measure theoretic arguments. However, if an another reduction theory can be patterned after Gelfand representation theorem it may give a considerable contribution to the theory of operator algebras. There have been many literatures in this direction as found in the recent comprehensive survey on representations of algebras by continuous sections by Hofmann [6].

Let $\mathfrak{A}$ be a von Neumann algebra with center 3. A basic idea in those discussions is to construct a suitable fibre algebra at each point of the spectrum $\Omega$ of 3 without the pathology of measure zero, and to consider a representation of $\mathfrak{A}$ as an algebra of cross-sections in this fibred space. For example, if we consider a minimal closed ideal $I(\omega)$ containing a point $\omega \in \Omega$, we get a quotient factorial $C^{*}$-algebra $\mathfrak{A} / I(\omega)$ and a representation of $\mathfrak{A}$ as a $C^{*}$-algebra of continuous cross-sections, or of continuous operator fields ([4], [14]). A peculier situation in a von Neumann algebra, however, is that there is a one-to-one correspondence $\omega \leftrightarrow \mathfrak{M}_{\omega}$ between the spectrum $\Omega$ and the maximal ideal space of $\mathfrak{A}$. The correspondence can be also considered for the commutant $\mathfrak{X}^{\prime}$ on the same space $\Omega$, so there would be a representation theory taking this advantage for von Neumann algebras. The situation has been exploited extensively by Teleman [12] in his algebraic reduction theory of a von Neumann algebra by using sheaf theory

In the following we shall propose a reduction theory of a finite von Neumann algebra following the idea in the Sakai's exposition [7] and Vesterstrøm [16]. We set the $C^{*}$-algebra $\mathfrak{A}(\omega)=\mathfrak{X} / \mathfrak{M}_{\omega}$ at $\omega \in \Omega$ as the fibre and ask further the algebra $\mathfrak{A}(\omega)$ to be a von Neumann algebra. 
Since this can not be expected in general as shown in Takemoto [9] and [10], we naturally assume $\mathfrak{A}$ to be a finite von Neumann algebra. In this case, the maximal ideal $\mathfrak{M}_{\omega}$ is particularly determined by $\boxminus$-application and the algebra $\mathfrak{A}(\omega)$ turns out to be a finite factor. On the other hand, one notable property of a von Neumann algebra among $C^{*}$-algebras is that it has the duality $\mathfrak{A}=\left(\mathfrak{A}_{*}\right)^{*}$, the conjugate space of the predual $\mathfrak{A}_{*}$. We mainly take this duality into our arguments, considering representations of $\mathfrak{A}$ and $\mathfrak{A}_{*}$ as an algebra of bounded weakly continuous fields over $\mathfrak{A}(\omega)^{\prime} s$ and as Banach spaces of functional fields over $\mathfrak{A}(\omega)_{*}^{\prime} s$. We start first with a von Neumann subalgebra $\mathscr{A}$ of 3 and the conditional expectation $\varepsilon$ to $\mathscr{A}$. By means of this $\varepsilon$ we define an ideal $\mathfrak{M}_{\omega}$ to each point $\omega$ of the spectrum $\Omega$ of $\mathscr{A}$ to the effect that the quotient algebra $\mathfrak{A}(\omega)=\mathfrak{A} / \mathfrak{M}_{\omega}$ becomes a finite von Neumann algebra. We shall introduce a certain Banach space of $\sigma$-weakly continuous $\mathscr{A}$-module mappings to $\mathscr{A}$ (the module predual of $\mathfrak{A}$ ), which turns out to be the space of all continuous fields over $\left\{\mathfrak{A}(\omega)_{*} ; \omega \in \Omega\right\}$ (Proposition 2.4). The algebra $\mathfrak{A}$ will be represented as an algebra of all bounded weakly continuous fields over $\left\{\mathfrak{T}(\omega), \mathfrak{R}(\omega)_{*} ; \omega \in \Omega\right\}$ under the duality between two spaces of cross-sections (Theorem 3.3). This could be regarded as a Gelfand type representation of $\mathfrak{H}$. In $\S 4$ we shall treat the reduction of subalgebras of $\mathfrak{A}$. The result of Takesaki [11] will be proved from our view point as one of consequences. The situation will, then, be clarified further in Theorem 4.8 giving a necessary and sufficient condition that $\widetilde{\mathfrak{B}(\omega)}=\tilde{\mathfrak{B}}(\omega)$ holds for a point $\omega \in \Omega$, where the notations mean the weak closure of $\mathfrak{B}(\omega)$ and $\mathfrak{B}$ in $\mathfrak{A}(\omega)$ and $\mathfrak{A}$ for a $C^{*}$-subalgebra $\mathfrak{B}$. In the final $\S 6$ we give a connection between $\sigma$-weakly continuous $\mathscr{A}$-module mappings and those elements in the module predual of $\Re$.

1. Preliminaries. In this section we provide some notations and facts that will be used later. We assume, throughout our discussions, $\mathfrak{A}$ to be a $\sigma$-finite von Neumann algebra. But the reader will notice that the assumption is merely used for convenience and the proofs can be mostly effected without this assumption. We shall be concerned with a von Neumann subalgebra $\mathscr{A}$ in the center 3 of $\mathscr{A}$. Let $\Omega$ be the spectrum of $\mathscr{A}$. We identify $\mathscr{A}$ with $C(\Omega)$, the space of all complex-valued continuous functions on $\Omega$. Let $\tau$ be a faithful normal trace of $\mathfrak{A}$. We fix this trace $\tau$ throughout our discussion. Then, it is known (cf. [15]) that there exists a unique faithful normal projection $\varepsilon$ of norm one to $\mathscr{A}$ invariant by $\tau$, called the conditional expectation to $\mathscr{A}$. The projection $\varepsilon$ satisfies, as in the case of $\boxminus$-application to the center, the equality $\varepsilon\left(x^{*} x\right)=\varepsilon\left(x x^{*}\right)$ 
for every $x \in \mathfrak{A}$. In fact, for every $x \in \mathfrak{A}$ and $a \in \mathscr{A}$, we have

$$
\begin{aligned}
& \left\langle a \varepsilon\left(x^{*} x\right), \tau\right\rangle=\left\langle\varepsilon\left(a x^{*} x\right), \tau\right\rangle \\
& \quad=\left\langle a x^{*} x, \tau\right\rangle=\left\langle x a x^{*}, \tau\right\rangle \\
& \quad=\left\langle a x x^{*}, \tau\right\rangle=\left\langle a \varepsilon\left(x x^{*}\right), \tau\right\rangle,
\end{aligned}
$$

and

$$
\left\langle a\left(\varepsilon\left(x^{*} x\right)-\varepsilon\left(x x^{*}\right)\right), \tau\right\rangle=0 .
$$

Hence $\varepsilon\left(x^{*} x\right)=\varepsilon\left(x x^{*}\right)$.

Let $\mathscr{L}(\mathfrak{A}, \mathscr{A})$ be the Banach space of all bounded linear mappings of $\mathfrak{A}$ into $\mathscr{A}$ with uniform norm. By an $\mathscr{A}$-module mapping, we mean a mapping $\Phi$ in $\mathscr{L}(\mathfrak{A}, \mathscr{A})$ such that $\Phi(a x b)=a \Phi(x) b$ for every $a, b \in \mathscr{A}$. We define the mapping $\varepsilon_{a}(a \in \mathfrak{A})$ by $\varepsilon_{a}(x)=\varepsilon(a x)=\varepsilon(x a)$, then $\varepsilon_{a}$ is a $\sigma$ weakly continuous $\mathscr{A}$-module mapping to $\mathscr{A}$. Let $V_{\mathfrak{a}}$ be the closure of $\left\{\varepsilon_{a} ; a \in \mathfrak{A}\right\}$ in $\mathscr{L}(\mathfrak{A}, \mathscr{A})$. This is a closed invariant subspace of $\mathscr{L}(\mathfrak{A}, \mathscr{A})$ in the following sense. For each $a \in \mathfrak{A}$ we consider linear transformations $L_{a}$ and $R_{a}$ on $\mathscr{L}(\mathfrak{A}, \mathscr{A})$ defined by $L_{a} \Phi(x)=\Phi(a x), R_{a} \Phi(x)=\Phi(x a)$. Then

$$
L_{a} V_{\mathfrak{x}} \subset V_{\mathfrak{x}} \text { and } R_{a} V_{\mathfrak{x}} \subset V_{\mathfrak{x}} \text { for every } a \in \mathfrak{X} \text {. }
$$

In particular, for a function $f \in \mathscr{A}, L_{f} \Phi(x)=R_{f} \Phi(x)=\Phi(f x)=f \Phi(x)$. Hence we understand by $f \circ \Phi$ the product of $f$ and $\Phi$ and in this sense $V_{\mathrm{a}}$ is an $\mathscr{A}$-module. Let $\omega$ be a point of $\Omega$. The set of all $x \in \mathfrak{A}$ with $\varepsilon\left(x^{*} x\right)(\omega)=0$ forms a closed ideal $\mathfrak{M}_{\omega}$ of $\mathfrak{A}$. When $\mathscr{A}=\mathfrak{Z}, \varepsilon$ coincides the $\boxminus$-application and $\mathfrak{M}_{\omega}$ becomes a maximal ideal. Let $\mathfrak{A}(\omega)$ be the quotient $C^{*}$-algebra of $\mathfrak{A}$ by $\mathfrak{M}_{\omega}$. The quotient homomorphism will be denoted by $\pi_{\omega}$ and the image of $x$ by $x(\omega)$. Let $\Phi$ be a bounded $\mathscr{A}$-module mapping to $\mathscr{A}$ such that $\Phi\left(\mathfrak{M}_{\omega}\right) \subset \mathfrak{M}_{\omega} \cap \mathscr{A}$. Then, $\Phi(x)(\omega)$ only depends on the image $x(\omega)$ for each $x \in \mathfrak{A}$ and $|\Phi(x)(\omega)| \leqq\|\Phi\| \cdot\|x(\omega)\|$. Hence $\Phi$ gives rise to a bounded functional $\Phi(\omega)$ on $\mathfrak{U}(\omega)$ by $\langle x(\omega), \Phi(\omega)\rangle=\Phi(x)(\omega)$. In particular, all elements in $V_{\mathfrak{a}}$ induce bounded functionals on $\mathfrak{A}(\omega)$ at each point $\omega$. Put $V_{\mathfrak{r}}(\omega)=\left\{\Phi(\omega) ; \Phi \in V_{\mathfrak{r}}\right\} . \quad V_{\mathfrak{r}}(\omega)$ is an invariant subspace of the conjugate space $\mathfrak{A}(\omega)^{*}$, that is, for every $\Phi \in V_{\mathfrak{q}}, R_{a(\omega)} L_{b(\omega)} \Phi(\omega)$ belongs again to $V_{\mathfrak{u}}(\omega)$ where

$$
\left\langle x(\omega), R_{a(\omega)} L_{b(\omega)} \Phi(\omega)\right\rangle=\langle b(\omega) x(\omega) a(\omega), \Phi(\omega)\rangle .
$$

It will be shown that $V_{\mathfrak{x}}(\omega)$ is even closed in $\mathfrak{A}(\omega)^{*}$ and $\mathfrak{A}(\omega)$ becomes the dual space of $V_{\mathfrak{x}}(\omega)$. We shall realize $V_{\mathfrak{x}}$ as the space of all continuous fields over $\left\{V_{\mathfrak{q}}(\omega)\right\}$. Hence, our first concern is to show the continuity of the function $\omega \rightarrow\|\Phi(\omega)\|$ for $\Phi \in V_{\mathfrak{r}}$. Here our case is a little different from those cases treated in [7] and [16] but the proof 
proceeds along the same line as in them. We only provide the whole proof of the key lemma.

Lemma 1.1. Let $\Phi$ be a $\sigma$-weakly continuous $\mathscr{A}$-module mapping to $\mathscr{A}$ such that $\Phi\left(\mathfrak{M}_{\omega}\right) \subset \mathfrak{M}_{\omega} \cap \mathscr{A}$ for each $\omega \in \Omega$. Then there exists a unitary element $u \in \mathfrak{R}$ such that $\|\Phi(\omega)\|=\Phi(u)(\omega)$. Hence the function $\omega \rightarrow\|\Phi(\omega)\|$ is continuous on $\Omega$.

PROoF. Let $\phi$ be a faithful normal state of $\mathscr{A}$ and put $\psi=\phi \circ \Phi$. Then $\psi$ is a $\sigma$-weakly continuous linear functional on $\mathfrak{A}$ and, since $\mathfrak{A}$ is finite, there exists a unitary element $u$ with $\langle u, \psi\rangle=\|\psi\|$. Therefore,

$$
\left\langle 1, R_{u} \psi\right\rangle=\|\psi\|=\left\|R_{u} \psi\right\|
$$

and $R_{u} \psi$ is a positive functional on $\mathfrak{R}$. We assert that $R_{u} \Phi$ is a positive mapping. In fact, let $x$ be a positive element and write real and imaginary parts of $\Phi(x u)$ into their positive and negative parts;

$$
\Phi(x u)=h^{+}-h^{-}+i\left(k^{+}-k^{-}\right) .
$$

We can find a projection $p$ of $\mathscr{A}$ such that $p k^{+}=k^{+}$and $p k^{-}=0$. Then

$$
\begin{aligned}
0 & \leqq\langle\Phi(p x u), \phi\rangle=\langle p \Phi(x u), \phi\rangle \\
& =\left\langle p\left(h^{+}-h^{-}\right), \phi\right\rangle+i\left\langle k^{+}, \phi\right\rangle .
\end{aligned}
$$

Hence $\left\langle k^{+}, \phi\right\rangle=0$ and $k^{+}=0$. Similarly $k^{-}=0$ and also we get $h^{-}=0$. Thus $\Phi(x u) \geqq 0$. Therefore, the functional $R_{u(\omega)} \Phi(\omega)$ becomes positive. Since $u(\omega)$ is a unitary element in $\mathfrak{H}(\omega)$, we have;

$$
\begin{aligned}
\|\Phi(\omega)\| & =\left\|R_{u(\omega)} \Phi(\omega)\right\|=\left\langle 1(\omega), R_{u(\omega)} \Phi(\omega)\right\rangle \\
& =\langle u(\omega), \Phi(\omega)\rangle=\Phi(u)(\omega) \text { for each } \omega \in \Omega .
\end{aligned}
$$

This completes the proof.

We notice that $\|\Phi\|=\sup \{\|\Phi(\omega)\| ; \omega \in \Omega\}$ for such a mapping. In fact, we have $\|\Phi(\omega)\| \leqq\|\Phi\|$ and for each $x \in \mathfrak{A}$ with $\|x\| \leqq 1$,

$$
\begin{aligned}
\|\Phi(x)\| & =\sup \{|\Phi(x)(\omega)| ; \omega \in \Omega\} \\
& =\sup \{|\langle x(\omega), \Phi(\omega)\rangle| ; \omega \in \Omega\} \\
& \leqq \sup \{\|\Phi(\omega)\| ; \omega \in \Omega\} .
\end{aligned}
$$

2. Continuous functional fields over preduals. Let $S$ be a closed subset of $\Omega$ and put $J=\left\{x \in \mathfrak{A} ; \varepsilon\left(x^{*} x\right)(\omega)=0\right.$ for every $\left.\omega \in S\right\} . \quad J$ is a closed ideal of $\mathscr{A}$. Let $I=\mathscr{A} \cap J$, then $I$ is a closed ideal of $\mathscr{A}$. By the definition of $V_{\mathfrak{x}}, \Phi(J) \subset I$ for every $\Phi \in V_{\mathfrak{q}}$. Hence the induced mapping $\Phi(S)$ from $\mathfrak{A} / J \equiv \mathfrak{A}(S)$ to $\mathscr{A} / I \equiv \mathscr{A}(S)$ is defined by $\Phi(S)(x(S))=\Phi(x)(S)$ where $x(S)$ means the image of $x$ in $\mathfrak{T}(S)$. Let $V_{\mathfrak{r}}(S)=\left\{\Phi(S) ; \Phi \in V_{\mathrm{r}}\right\}$, 
then $V_{\mathfrak{r}}(S)$ is an invariant subspace of $\mathscr{L}(\mathfrak{A}(S), \mathscr{A}(S))$. The following lemma is a slight modification of Proposition 1.3 in [16]. We omit the proof.

LEMmA 2.1. For each $\Phi \in V_{\mathfrak{r}}$,

(i) $\Phi(S)$ is norm bounded and $\|\Phi(S)\|=\sup \{\|\Phi(\omega)\| ; \omega \in S)$. Moreover, $\|\Phi(S)\|$ coincides with the quotient norm in $V_{\mathfrak{x}} / K$ where $K$ is the kernel of the mapping $\Phi \rightarrow \Phi(S)$.

(ii) $V_{\mathfrak{q}}(S)$ formes a closed invariant subspace of $\mathscr{L}(\mathfrak{A}(S), \mathscr{A}(S))$.

In particular in case where $S=\{\omega\}, V_{\mathfrak{r}}(\omega)$ is a closed invariant subspace of $\mathfrak{A}(\omega)^{*}$. Another consequence of this lemma is that for any functional $\Phi(\omega)$ with $\|\Phi(\omega)\|<1$ there exists an element $\Phi_{0} \in V_{\text {a }}$ such that $\Phi_{0}(\omega)=\Phi(\omega)$ and $\left\|\Phi_{0}\right\|<1$. This fact will be often used later.

Now we can also prove the following generalization of the result of [7] and [16] following the lines of their arguments. We leave the proof to the reader.

Proposition 2.2. For each $\omega \in \Omega, \mathfrak{A}(\omega)$ is isomorphic $V_{\mathfrak{q}}(\omega)^{*}=$ $\mathfrak{U}(\omega)^{* *} / V_{\mathfrak{q}}(\omega)^{0}$ where $V_{\mathfrak{x}}(\omega)^{0}$ means the polar of $V_{\mathfrak{x}}(\omega)$ in $\mathfrak{A}(\omega)^{* *}$. Thus, $\mathfrak{U}(\omega)$ is a finite von Neumann algebra with the predual $V_{\mathfrak{x}}(\omega)$ and the faithful normal trace $\varepsilon(\omega)=\varepsilon_{1}(\omega)$.

From the above fact an element $\Phi$ in $V_{\mathfrak{x}}$ can be regarded as a functional field over preduals $V_{\mathfrak{x}}(\omega)^{\prime} s$. We denote this field by $\hat{\Phi}$ and put $\hat{V}_{\mathfrak{x}}=\left\{\widehat{\Phi} ; \Phi \in V_{\mathfrak{r}}\right\}$. We shall give the definition of a continuous field defined by $\left\{V_{\mathfrak{r}}(\omega), \hat{V}_{\mathfrak{r}}\right\}$.

DEFinition 2.3. A functional field $\Psi$ over $\left\{V_{\mathfrak{q}}(\omega)\right\}$ is said to be continuous with respect to $\hat{V}_{\mathrm{x}}$ iff, for each $\varepsilon>0$ and $\omega_{0} \in \Omega$, there exist an element $\hat{\Phi} \in \hat{V}_{\mathfrak{x}}$ and a neighborhood $U\left(\omega_{0}\right)$ of $\omega_{0}$ such that $\|\Phi(\omega)-\Psi(\omega)\|<\varepsilon$ for every $\omega \in U\left(\omega_{0}\right)$.

Let $C\left(\Omega, V_{\mathfrak{r}}(\omega), \hat{V}_{\mathfrak{r}}\right)$ be the set of all continuous fields. Then, the function $\omega \rightarrow\|\Psi(\omega)\|$ is continuous for each $\Psi \in C\left(\Omega, V_{\mathfrak{n}}(\omega), \hat{V}_{\mathfrak{n}}\right)$ and we define the norm $\|\Psi\|=\max \{\|\Psi(\omega)\| ; \omega \in \Omega\}$. It is known (cf. [2], [14]) that with this norm $C\left(\Omega, V_{\mathfrak{q}}(\omega), \hat{V}_{\mathfrak{q}}\right)$ becomes an $\mathscr{A}$-module

The following proposition is rather a standard result in the theory of continuous fields.

Proposition 2.4. The correspondence $\Phi \leftrightarrow \hat{\Phi}$ gives an isometry between $V$ and $C\left(\Omega, V_{\mathfrak{r l}}(\omega), \hat{V}_{\mathfrak{r}}\right)$.

Proof. By the remark after Lemma 1.1, the correspondence gives an isometry from $V$ into $C\left(\Omega, V_{\mathfrak{r}}(\omega), \hat{V}_{\mathfrak{r}}\right)$. We assert that the isometry 
is onto. Take a continuous field $\Psi$ and a positive number $\varepsilon$. By the definition of continuity there exists a family $\left\{\left(U(\omega), \widehat{\Phi}_{\omega}\right) ; \omega \in \Omega\right\}$ of pairs of closed and open neighborhoods and elements of $\hat{V}_{\mathfrak{q}}$ such that $\| \Psi(\eta)-$ $\Phi_{\omega}(\eta) \|<\varepsilon$ for every $\eta \in U(\omega)$. Since $\Omega$ is compact, there exists a finite subcovering $\left\{U\left(\omega_{i}\right) ; i=1,2, \cdots, n\right\}$ of $\{U(\omega) ; \omega \in \Omega\}$ and, as each $U(\omega)$ is closed and open, we may assume that $\left\{U\left(\omega_{i}\right)\right\}$ are mutually disjoint. Let $z_{i}$ be the projection in $\mathscr{A}$ corresponding to $U\left(\omega_{i}\right)$. Writing as $\hat{\Phi}=$ $\sum_{i=1}^{n} z_{i} \circ \widehat{\Phi}_{\omega_{i}}$, we see that $\hat{\Phi} \in \hat{V}_{\mathfrak{\Re}}$ and $\|\Psi(\omega)-\Phi(\omega)\|<\varepsilon$ for every $\omega \in \Omega$. Hence,

$$
\|\Psi-\hat{\Phi}\|=\sup \{\|\Psi(\omega)-\Phi(\omega)\| ; \omega \in \Omega\}<\varepsilon .
$$

As $\varepsilon$ is arbitrary, this means $\Psi \in \hat{V}_{\mathfrak{r}}$. This completes the proof.

From this proposition we see that each continuous field is realized as a module mapping in $V_{\mathfrak{x}}$.

It should be noticed that contrary to the present case, the space of all continuous field is usually quite bigger than the original induced family of fields. For example, $C(\Omega)$ can be considered as $C(\Omega, C(\omega), \widehat{C})$ where $C(\omega)$ is the fibre of complex numbers and $\hat{C}$ is the set of all constant functions.

3. Weakly continuous operator fields over von Neumann algebras and the Gelfand representation of $\mathfrak{A}$. An element $x \in \mathfrak{A}$ can be considered as an operator field over finite von Neumann algebras $\{\mathfrak{U}(\omega) ; \omega \in \Omega\}$. We denote this operator field by $\hat{x}$ and call this the Gelfand representation of $x$. Let $\hat{\mathfrak{A}}=\{\hat{x} ; x \in \mathfrak{A}\}$. We shall characterize those fields in $\hat{\mathfrak{A}}$ among operator fields over $\{\mathfrak{A}(\omega)\}$.

DEFINITION 3.1. An operator field $a$ over $\{\mathfrak{U}(\omega)\}$ is called a bounded weakly continuous field if the function $\omega \rightarrow\|a(\omega)\|$ is bounded and, for each $\Phi \in V_{\mathfrak{r}}$, the function $\omega \rightarrow\langle a(\omega), \Phi(\omega)\rangle$ is continuous on $\Omega$.

Let $W\left(\Omega, \mathfrak{R}(\omega), V_{\mathfrak{r}}(\omega)\right)$ be the space of all bounded weakly continuous fields. We consider in $W\left(\Omega, \mathfrak{R}(\omega), V_{\mathfrak{x}}(\omega)\right)$ the norm $\|a\|=\sup \{\|a(\omega)\| ; \omega \in \Omega\}$. Then, it is a straightforward calculation to see that the space is an $\mathscr{A}$ module Banach space. Let $\left[\tau \circ V_{\mathrm{x}}\right]$ be the closed subspace of $\mathfrak{A}_{*}$ spanned by $\left\{\tau \circ \Phi ; \Phi \in V_{\mathrm{x}}\right\}$. The space is invariant and total, that is, $\langle x, \phi\rangle=0$ for every $\phi \in\left[\tau \circ V_{\mathfrak{x}}\right]$ implies $x=0$. Hence $\mathfrak{A}_{*}=\left[\tau \circ V_{\mathrm{q}}\right]$. Let $\mu$ be the normal measure in $\Omega$ corresponding to the restriction of $\tau$ to $\mathscr{A}$. The next lemma can be proved following after the proof of Proposition 1.4 in [16]. We omit the detail.

Lemma 3.2. For each $\sigma$-weakly continuous $\mathscr{A}$-module mapping $\Phi$ to $\mathscr{A}$ such that $\Phi\left(\mathfrak{M}_{\omega}\right) \subset \mathfrak{M}_{\omega} \cap \mathscr{A}$, we have; 


$$
\|\tau \circ \Phi\|=\int_{\Omega}\|\Phi(\omega)\| d \mu .
$$

Now take an element $x \in \mathfrak{A}$, then for each $\Phi \in V_{\mathfrak{q}}$ we have

$$
\langle x(\omega), \Phi(\omega)\rangle=\Phi(x)(\omega) \text { and }\|x\|=\sup \{\|x(\omega)\| ; \omega \in \Omega\} \text {. }
$$

Hence $\hat{x}$ is a bounded weakly continuous field whose norm is equal to that of $x$. As we have named before this may be regarded as a noncommutative extension of the Gelfand representation of commutative $C^{*}$ algebras to the case of finite von Neumann algebras. In fact, we get the following representation theorem.

THEOREM 3.3. The representation $x \rightarrow \widehat{x}$ gives an isometry between $\mathfrak{X}$ and $W\left(\Omega, \mathfrak{A}(\omega), V_{\mathfrak{q}}(\omega)\right)$, that is, $\hat{\mathfrak{A}}=W\left(\Omega, \mathfrak{A}(\omega), V_{\mathfrak{r}}(\omega)\right)$.

Proof. It suffices to show that the correspondence is onto, so let $a$ be a bounded weakly continuous field over $\left\{\mathfrak{U}(\omega), V_{\mathfrak{q}}(\omega)\right\}$. For each $\Phi \in V_{\mathfrak{x}}$, we have

$$
\begin{aligned}
\int_{\Omega}|\langle a(\omega), \Phi(\omega)\rangle| d \mu & \leqq\|a\| \int_{\Omega}\|\Phi(\omega)\| d \mu \\
& =\|a\| \cdot\|\tau \circ \Phi\| .
\end{aligned}
$$

The last equality holds by Lemma 3.2. Since the correspondence $\Phi \rightarrow \tau \circ \Phi$ is one-to-one, we can consider a bounded linear functional,

$$
\tau \circ \Phi \rightarrow \int_{\Omega}\langle a(\omega), \Phi(\omega)\rangle d \mu,
$$

that can be extended to the space $\left[\tau \circ V_{\mathfrak{r}}\right]=\mathfrak{A}_{*}$. Therefore, there exists an element $\breve{a} \in \mathfrak{X}$ such that

$$
\langle\grave{a}, \tau \circ \Phi\rangle=\int_{\Omega}\langle a(\omega), \Phi(\omega)\rangle d \mu
$$

for every $\Phi \in V_{\mathfrak{a}}$. We assert that $\check{a}(\omega)=a(\omega)$ for every $\omega \in \Omega$. In fact, for each $f \in \mathscr{A}$ and $\Phi \in V_{\mathfrak{a}}, f \circ \Phi$ belongs to $V_{\mathfrak{a}}$ and hence

$$
\begin{aligned}
\int_{\Omega} f(\omega) & \langle\breve{a}(\omega)-a(\omega), \Phi(\omega)\rangle d \mu=\int_{\Omega}\langle\breve{a}(\omega)-a(\omega), f \circ \Phi(\omega)\rangle d \mu \\
& =\int_{\Omega}\langle\breve{a}(\omega), f \circ \Phi(\omega)\rangle d \mu-\int_{\Omega}\langle a(\omega), f \circ \Phi(\omega)\rangle d \mu \\
& =\int_{\Omega} f \circ \Phi(\breve{a})(\omega) d \mu-\langle\breve{a}, \tau \circ f \circ \Phi\rangle \\
& =\langle f \circ \Phi(\breve{a}), \tau\rangle-\langle f \circ \Phi(\widetilde{a}), \tau\rangle=0 .
\end{aligned}
$$

Therefore, 


$$
\langle\breve{a}(\omega)-a(\omega), \Phi(\omega)\rangle=0 \quad \mu \text {-a.e. } \omega \text {. }
$$

Since the above function is continuous, this implies

$$
\langle\breve{a}(\omega)-a(\omega), \Phi(\omega)\rangle=0 \text { for every } \omega \in \Omega .
$$

Thus, by Proposition 2.2, $\breve{a}(\omega)=a(\omega)$ for every $\omega \in \Omega$ and $\breve{a}$ is the corresponding element of $\mathfrak{A}$ to the field $a$. Moreover, we have $\|\check{a}\|=\|a\|$. This completes the proof.

Notice that, in case of $\mathfrak{X}=\mathscr{A}, V_{\mathfrak{R}}$ is nothing but the space $C(\Omega)$ itself considered as the space of mappings in $C(\Omega)$ by multiplications and the above representation is reduced to the usual Gelfand representation. Thus our treatment means that we are not concerned with the whole measure theoretic duality $L^{\infty}(\Omega, \mu)=L^{1}(\Omega, \mu)^{*}$ but are rather inclined to know the nuclear relation between their subspaces $(C(\Omega), C(\Omega))$ by considering the module duality in our fibred space. Essentially our discussion is a decomposition theory of the conditional expectation $\varepsilon$ into numerical normal traces. In particular, when $\mathscr{A}=3$, the expectation $\varepsilon$ is the $\sharp$ application and the fibre algebras $\mathfrak{U}(\omega)^{\prime} s$ become finite factors (unfortunately, mostly acting on non-separable Hilbert spaces). The decomposition of the trace $\tau$ invokes a measure theoretic reduction theory of finite von Neumann algebras, whereas the decomposition of the $甘$-application leads us to the present reduction theory of continuous type.

It is also to be noticed that the above representation is compatible with multiplication and *operation in the sense that the element $x y$ goes to the field composed by pointwise multiplication of $\hat{x}$ and $\hat{y}$ and $x^{*}$ goes to the field defined by $\left\{x(\omega)^{*}\right\}$. Therefore, in this case, $W\left(\Omega, \mathfrak{N}(\omega), V_{\mathfrak{A}}(\omega)\right)$ becomes a $C^{*}$-algebra by pointwise multiplication and *operation and the representation is actually a *-isomorphism. However, even if we give a functional field, the space of bounded weakly continuous field with respect to this functional field might not be closed with pointwise multiplication, that is, this space might not be an algebra.

It is known ([16; Theorem 3.4]) that, in general, one can not expect the continuity of the function $\omega \rightarrow\|x(\omega)\|$ for $x \in \mathfrak{A}$. However, as seen from Lemma 2.1 the function $\omega \rightarrow\|x(\omega)\|$ can be expressed as the supremum of a family of continuous function, $\left\{|\langle x(\omega), \Phi(\omega)\rangle|\right.$; $\left.\|\Phi\|<1, \Phi \in V_{\mathfrak{r}}\right\}$. Therefore, it is lower semicontinuous, and hence continuous except for a non-dense subset.

Let $V$ be the space of all $\sigma$-weakly continuous 3-module mapping from $\mathfrak{A}$ to 3. Halpern has proved ([5; Theorem 3]) that an arbitrary von Neumann algebra $\mathfrak{A}$ is expressed as the space of all bounded 3 -module mappings from $V$ to 3 , the module dual of $V$. In the present case where 
$\mathfrak{A}$ is a $\sigma$-finite von Neumann algebra, an element $x \in \mathfrak{A}$ gives rise to a bounded $\mathscr{A}$-module mapping $\theta_{x}$ from $V_{\mathfrak{r}}$ to $\mathscr{A}$ by defining $\theta_{x}(\Phi)=\Phi(x)$. Moreover, we have;

$$
\begin{aligned}
\left\|\theta_{x}\right\| & =\sup \left\{\left\|\theta_{x}(\Phi)\right\| ; \Phi \in V_{\mathfrak{r}},\|\Phi\|<1\right\} \\
& =\sup \left\{|\Phi(x)(\omega)| ; \omega \in \Omega, \Phi \in V_{\mathfrak{r}},\|\Phi\|<1\right\} \\
& =\sup \left\{|\langle x(\omega), \Phi(\omega)\rangle| ; \omega \in \Omega, \Phi(\omega) \in V_{\mathfrak{r}}(\omega),\|\Phi(\omega)\|<1\right\} \\
& =\sup \{\|x(\omega)\| ; \omega \in \Omega\}=\|x\| .
\end{aligned}
$$

That is, the correspondence $x \rightarrow \theta_{x}$ is an isometry. Therefore, we get the following more precise formulation of this kind of representation theorem.

THEOREM 3.4. Let $\mathfrak{A}$ be a $\sigma$-finite finite von Neumann algebra, then the above correspondence gives an isometry between $\mathfrak{A}$ and the space of all bounded $\mathscr{A}$-module mappings from $V_{\mathfrak{a}}$ to $\mathscr{A}$.

Proof. We must show that the correspondence is onto. Let $\theta$ be a bounded $\mathscr{A}$-module mapping from $V_{\mathfrak{x}}$ to $\mathscr{A}$. Take a point $\omega_{0} \in \Omega$ and fix. We shall show that $\theta$ gives rise to a bounded linear functional $\theta\left(\omega_{0}\right)$ on $V_{\mathfrak{r}}\left(\omega_{0}\right)$. Let $\Phi$ be an element of $V_{\mathfrak{r}}$. Then $\theta(\Phi)\left(\omega_{0}\right)$ depends only on the functional $\Phi\left(\omega_{0}\right)$. In fact, consider an another element $\Psi \in V_{\mathfrak{x}}$ such as $\Psi\left(\omega_{0}\right)=\Phi\left(\omega_{0}\right)$. Then, for any positive number $\varepsilon$ there exists a closed and open neighborhood $U$ of $\omega_{0}$ such that

$$
\|\Psi(\omega)-\Phi(\omega)\|<\varepsilon /\|\theta\| \text { for every } \omega \in U \text {. }
$$

Let $z$ be a corresponding projection of $\mathscr{A}$ to $U$. We have,

$$
\|z(\Phi-\Psi)\|=\|z(\hat{\Phi}-\hat{\Psi})\| \leqq \varepsilon /\|\theta\| \text { • }
$$

Hence,

$$
\begin{aligned}
& \left|\theta(\Phi)\left(\omega_{0}\right)-\theta(\Psi)\left(\omega_{0}\right)\right|=\left|z\left(\omega_{0}\right) \theta(\Phi-\Psi)\left(\omega_{0}\right)\right| \\
& \quad=\left|\theta(z \circ(\Phi-\Psi))\left(\omega_{0}\right)\right| \leqq\|\theta\| \cdot\|z(\Phi-\Psi)\| \leqq \varepsilon .
\end{aligned}
$$

As $\varepsilon$ is arbitrary, $\theta(\Phi)\left(\omega_{0}\right)=\theta(\Psi)\left(\omega_{0}\right)$. We define the linear functional $\theta\left(\omega_{0}\right)$ by $\left\langle\theta\left(\omega_{0}\right), \Phi\left(\omega_{0}\right)\right\rangle=\theta(\Phi)\left(\omega_{0}\right)$. By Lemma 2.1, $\theta\left(\omega_{0}\right)$ is bounded and $\left\|\theta\left(\omega_{0}\right)\right\| \leqq\|\theta\|$, hence $\theta(\omega) \in \mathfrak{A}(\omega)$. Clearly the field $\omega \rightarrow \theta(\omega)$ is a bounded weakly continuous field over $\left\{\mathfrak{U}(\omega), V_{\mathfrak{x}}(\omega)\right\}$. Hence, by Theorem 3.3, there is an element $x \in \mathfrak{X}$ such that $x(\omega)=\theta(\omega)$ for every $\omega \in \Omega$. Thus,

and $\theta_{x}(\Phi)=\theta(\Phi)$ for every $\Phi \in V_{\mathfrak{x}}$.

$$
\begin{aligned}
\theta_{x}(\Phi)(\omega) & =\Phi(x)(\omega)=\langle x(\omega), \Phi(\omega)\rangle \\
& =\langle\theta(\omega), \Phi(\omega)\rangle=\theta(\Phi)(\omega)
\end{aligned}
$$

Henceforth we call $V_{\mathfrak{x}}$ the module predual of $\mathfrak{A}$. 
4. Restricted functional fields to subalgebras. Let $\mathfrak{B}$ be a $C^{*}$ subalgebra of $\mathfrak{A}$. We assume always $\mathfrak{B}$ contains the unit of $\mathfrak{A}$. We mean by $\mathfrak{B}(\omega)$ the quotient image of $\mathfrak{B}$ in $\mathfrak{A}(\omega)$. $\mathfrak{B}(\omega)$ is a $C^{*}$-subalgebra of $\mathfrak{A}(\omega)$. We denote by $\left.\Phi\right|_{\mathfrak{\vartheta}}$ and $\left.\Phi(\omega)\right|_{\mathscr{\vartheta}(\omega)}$ the restrictions of $\Phi$ and $\Phi(\omega)$ to $\mathfrak{B}$ and $\mathfrak{B}(\omega)$, respectively. In $\S 1$, we have noted that the equality $\|\Phi\|=\sup \{\|\Phi(\omega)\| ; \omega \in \Omega\}$ holds for a bounded $\mathscr{A}$-module mapping $\Phi$ to $\mathscr{A}$ such as $\Phi\left(\mathfrak{M}_{\omega}\right) \subset \mathfrak{M}_{\omega} \cap \mathscr{A}$. However, the calculation also goes through the same way in this case of restricted mappings and we get

Lemma 4.1. Let $\mathfrak{B}$ be a $C^{*}$-subalgebra of $\mathfrak{X}$, then

$$
\left\|\left.\Phi\right|_{\left.\right|_{\mathfrak{B}}}\right\|=\sup \left\{\left\|\left.\Phi(\omega)\right|_{\mathscr{\vartheta}(\omega)}\right\| ; \omega \in \Omega\right\} .
$$

The following lemma should be due to Theorem 7 in Dixmier [1; Chapter I, §6], once one notices that the functional $\langle x(\omega), \varepsilon(\omega)\rangle=\varepsilon(x)(\omega)$ is a normal trace of $\mathfrak{A}(\omega)$. We omit the detail.

Lemma 4.2. For the conditional expectation $\varepsilon$ of $\mathfrak{A}$ to $\mathscr{A}$, we have

$$
|\varepsilon(a b)| \leqq \varepsilon(|a b|) \leqq\|a\| \varepsilon(|b|)
$$

for every $a, b \in \mathfrak{A}$ where $|a|$ means the absolute value of an operator a.

As a consequence of this Lemma, we get the following

Lemma 4.3. Let $\mathfrak{B}$ be a $C^{*}$-subalgebra of $\mathfrak{A}$. Then, for each $a \in \mathfrak{B}$, we have;

$$
\left\|\left.\varepsilon_{a}(\omega)\right|_{\mathscr{B}(\omega)}\right\|=\varepsilon(|a|)(\omega) \quad \text { for } \quad \omega \in \Omega .
$$

Hence,

$$
\left\|\left.\varepsilon_{a}\right|_{\mathscr{B}}\right\|=\|\varepsilon(|a|)\| \text {. }
$$

Proof. By the above Lemma, we have, for each $x \in \mathfrak{B}$,

$$
\begin{aligned}
\left|\left\langle x(\omega), \varepsilon_{a}(\omega)\right\rangle\right| & =\left|\varepsilon_{a}(x)(\omega)\right|=|\varepsilon(a x)(\omega)| \\
& =|\varepsilon(x a)|(\omega) \leqq|| x \| \varepsilon(|a|)(\omega) .
\end{aligned}
$$

Hence,

$$
\left\|\left.\varepsilon_{a}(\omega)\right|_{\mathscr{B}(\omega)}\right\| \leqq \varepsilon(|a|)(\omega) .
$$

Let $\widetilde{B}(\omega)$ be the weak closure of $\mathfrak{B}(\omega)$ in $\mathfrak{A}(\omega)$, and let $a(\omega)=u|a(\omega)|$ be the polar decomposition of $a(\omega)$. The element $|a(\omega)|=|a|(\omega)$ belongs to $\mathfrak{B}(\omega)$ and $u \in \overparen{\mathfrak{B}(\omega)}$. Hence,

$$
\begin{aligned}
\varepsilon(|a|)(\omega) & =\langle|a(\omega)|, \varepsilon(\omega)\rangle=\left\langle u^{*} a(\omega), \varepsilon(\omega)\right\rangle \\
& =\left\langle u^{*}, R_{a(\omega)} \varepsilon(\omega)\right\rangle=\left\langle u^{*}, \varepsilon_{a}(\omega)\right\rangle \\
& \leqq\left\|\left.\varepsilon_{a}(\omega)\right|_{\mathscr{\vartheta}(\omega)}\right\|=\left\|\left.\varepsilon_{a}(\omega)\right|_{\mathscr{\vartheta}(\omega)}\right\| .
\end{aligned}
$$


Therefore,

$$
\varepsilon(|a|)(\omega)=\left\|\left.\varepsilon_{a}(\omega)\right|_{\mathscr{B}(\omega)}\right\| \text { for every } \omega \in \Omega .
$$

By Lemma 4.1,

$$
\left\|\left.\varepsilon_{a}\right|_{\mathscr{\vartheta}}\right\|=\sup \{\varepsilon(|a|)(\omega) ; \omega \in \Omega\}=\|\varepsilon(|a|)\| \cdot
$$

Now let $\mathfrak{B}$ be a von Neumann subalgebra of $\mathfrak{A}$ containing $\mathscr{A}$. Then $\mathfrak{B}$ itself is a $\sigma$-finite finite von Neumann algebra whose center contains $\mathscr{A}$. Hence one can construct the module predual of $\mathfrak{B}$ as we have done for $\mathfrak{A}$. Let $\lambda$ be the restriction of $\varepsilon$ to $\mathfrak{B}$, then $\lambda$ is the conditional expectation to $\mathscr{A}$ with respect to the trace $\left.\tau\right|_{\mathscr{B}}$, the restriction of $\tau$ to $\mathfrak{B}$. Let $V_{\mathfrak{B}}$ be the uniform closure of $\left\{\lambda_{a} ; a \in \mathfrak{B}\right\}$ in $\mathscr{L}(\mathfrak{B}, \mathscr{A})$. The following result corresponds to the fact for usual predual spaces, that is, the fact $\left.\mathfrak{A}_{*}\right|_{\mathbb{B}}=\mathfrak{B}_{*}$.

THeOREM 4.4. Let $\mathfrak{B}$ be a von Neumann subalgebra containing $\mathscr{A}$, then $V_{\mathfrak{B}}=\left.V_{\mathfrak{x}}\right|_{\mathfrak{B}}=\left.\left\{\left.\Phi\right|_{\mathfrak{y}} ; \Phi \in V_{\mathfrak{x}}\right\}_{\text {. Hence }} V_{\mathfrak{x}}\right|_{\mathfrak{B}}$ is a closed invariant subspace of $\mathscr{L}(\mathfrak{B}, \mathscr{A})$.

Proof. Let $\delta$ be the conditional expectation to $\mathfrak{B}$ with respect to $\tau$, that is, $\delta$ is a unique faithful normal projection to $\mathfrak{B}$ invariant by $\tau$. By the uniqueness, we have $\varepsilon=\lambda \circ \delta$. Take an element $\varepsilon_{a}$ in $V_{\mathfrak{x}}$. Then, for $x \in \mathfrak{B}$,

$$
\begin{aligned}
\varepsilon_{a}(x) & =\varepsilon(a x)=\lambda \circ \delta(a x)=\lambda(\delta(a) x) \\
& =\lambda_{\delta(a)}(x) .
\end{aligned}
$$

Hence, $\left.\varepsilon_{a}\right|_{\mathfrak{y}}=\lambda_{\tilde{\partial}(a)} \in V_{\mathfrak{y}}$ and $\left.V_{\mathfrak{x}}\right|_{\mathfrak{\Re}} \subset V_{\mathfrak{y}}$.

Conversely, take an element $\Psi \in V_{\mathfrak{*}}$. Then the mapping $\Psi \circ \delta$ belongs to $\mathscr{L}(\mathfrak{A}, \mathscr{A})$ and $\|\Psi \circ \delta\|=\|\Psi\|$. Let $\left\{a_{n}\right\}$ be a sequence in $\mathfrak{B}$ such that $\left\|\lambda_{a_{n}}-\Psi\right\| \rightarrow 0$. Put $\Phi_{n}=\lambda_{a_{n}} \circ \delta$ and $\Phi=\Psi \circ \delta$ We have, for $x \in \mathfrak{A}$,

$$
\begin{aligned}
\Phi_{n}(x) & =\lambda_{a_{n}} \circ \delta(x)=\lambda\left(a_{n} \delta(x)\right)=\lambda \circ \delta\left(a_{n} x\right) \\
& =\varepsilon_{a_{n}}(x) \text { and } \Phi_{n} \in V_{\mathfrak{q}} .
\end{aligned}
$$

Moreover,

$$
\left\|\Phi_{n}-\Phi\right\|=\left\|\left(\lambda_{a_{n}}-\Psi\right) \circ \delta\right\|=\left\|\lambda_{a_{n}}-\Psi\right\| \rightarrow 0 .
$$

Therefore, $\Phi \in V_{\mathfrak{r}}$ and $\Psi=\left.\left.\Phi\right|_{\mathfrak{\Re}} \in V_{\mathfrak{r}}\right|_{\mathfrak{g}}$.

A consequence of this theorem is the following proof of Takesaki's theorem in terms of fields of algebras and their preduals.

THEOREM 4.5 (Takesaki [11]). Let $\mathfrak{B}$ be a von Neumann subalgebra 
of $\mathfrak{A}$ containing $\mathscr{A}$. Then, for each $\omega \in \Omega, \mathfrak{B}(\omega)$ is a von Neumann subalgebra of $\mathfrak{A}(\omega)$.

Proof. Let $V_{\mathfrak{g}}(\omega)$ be the fibre induced from $V_{\Re}$. Then, $V_{\mathfrak{g}}(\omega)$ is the predual of the von Neumann algebra $\mathfrak{B} / \mathfrak{B} \cap \mathfrak{M}_{\omega}$. Let $\theta$ be the *isomorphism between $\mathfrak{B}(\omega)$ and $\mathfrak{B} / \mathfrak{B} \cap \mathfrak{M}_{\omega}$, then Theorem 4.4 shows that ${ }^{t} \theta$ induces an isometry between $V_{\mathfrak{y}}(\omega)$ and the quotient space $V_{\mathfrak{x}}(\omega) / \mathfrak{B}(\omega)^{\circ}$ where $\mathfrak{B}(\omega)^{\circ}$ means the polar of $\mathfrak{B}(\omega)$ in $V_{\mathfrak{x}}(\omega)$. Since $\left(V_{\mathfrak{x}}(\omega) / \mathfrak{B}(\omega)^{\circ}\right)^{*}=$ $\widetilde{\mathfrak{B}(\omega)}$, the weak closure of $\mathfrak{B}(\omega)$, we have $\theta^{-1}\left(\mathfrak{B} / \mathfrak{B} \cap \mathfrak{M}_{\omega}\right)=\overparen{\mathfrak{B}(\omega)}=\mathfrak{B}(\omega)$.

Combining this with Theorem 3.3 we have

CoRollary 4.6. Let $\mathfrak{B}$ be a von Neumann subalgebra of $\mathfrak{A}$ containing $\mathscr{A}$, then $\mathfrak{B}$ can be considered as the set of all bounded weakly continuous fields ranging over $\mathfrak{B}(\omega)^{\prime}$ s.

It has been pointed out in [17] that in every quotient image of a von Neumann algebra the image of the center coincides with the center of the quotient algebra. Therefore, each $3(\omega)$ is the center of $\mathfrak{A}(\omega)$, hence a von Neumann subalgebra. Let $\boxminus$ be the $\boxminus$-application of $\mathfrak{A}$, that is, the unique faithful normal expectation to 3 such that $\boxminus\left(x^{*} x\right)=\Xi\left(x x^{*}\right)$.

Proposition 4.7. The center 3 of $\mathfrak{A}$ can be considered as the set of all bounded weakly continuous fields ranging over $3(\omega)^{\prime}$ s. Furthermore, the mapping $\boxminus(\omega): x(\omega) \rightarrow \exists(x)(\omega)$ is the $\boxminus$-application of the finite von Neumann algebra $\mathfrak{A}(\omega)$.

Proof. As $\varepsilon$ is the unique $\tau$-invariant conditional expectation, we have $\varepsilon=\varepsilon_{1} \circ$ where $\varepsilon_{1}$ is the restriction of $\varepsilon$ to 3. Suppose $x(\omega)=0$, then $\varepsilon\left(x^{*} x\right)(\omega)=0$. Hence

$$
\varepsilon_{1} \circ \models\left(x^{*} x\right)(\omega) \geqq \varepsilon_{1}\left(\xi(x)^{*} \boxminus(x)\right)(\omega)=0,
$$

and $\boxminus(x)(\omega)=0$. Thus $\xi(\omega)$ is well defined and one easily sees that $\boxminus(\omega)$ is a projection of norm one from $\mathfrak{N}(\omega)$ to $3(\omega)$ such as $\boxminus(\omega)\left(x^{*}(\omega) x(\omega)\right)=$ $\xi(\omega)\left(x(\omega) x^{*}(\omega)\right)$. Therefore, $\xi(\omega)$ is the $\boxminus$-application of $\mathfrak{A}(\omega)$ (cf. [1; Chapter III, 8]).

Now Theorem 4.4 and Lemma 1.1 show that the function $\omega \rightarrow$ $\left\|\left.\Phi(\omega)\right|_{\mathfrak{B}(\omega)}\right\|$ is continuous for each $\Phi \in V_{\mathfrak{x}}$ for such a von Neumann subalgebra $\mathfrak{B}$ as $\mathfrak{B} \supset \mathscr{A}$. And Corollary 4.5 says that in this case the quotient image of $\mathfrak{B}$ is weakly closed in $\mathfrak{A}(\omega)$. The next theorem will clarify this situation. Let $\tilde{\mathfrak{B}}$ be the weak closure of $\mathfrak{B}$.

THeorem 4.8. Let $\mathfrak{B}$ be a $C^{*}$-subalgebra of $\mathfrak{A}$ containing $\mathscr{A}$. Then $\widetilde{\mathfrak{B}\left(w_{0}\right)}=\tilde{\mathfrak{B}}\left(\omega_{0}\right)$ for a point $\omega_{0} \in \Omega$ if and only if, for each $\Phi \in V_{\mathfrak{x}}$, the function $\omega \rightarrow\left\|\left.\Phi(\omega)\right|_{\mathscr{B}(\omega)}\right\|$ is continuous at $\omega_{0}$. 
That is, the continuity of restriction functional fields covers a weak point of the discontinuity of the quotient homomorphism $\pi_{\omega}$.

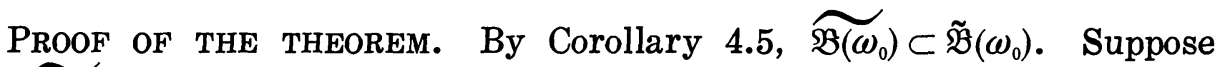

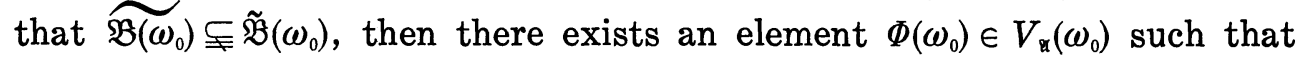

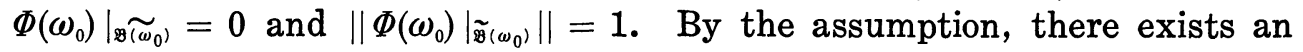

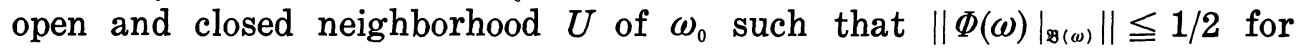
$\omega \in U$. Let $z$ be the characteristic function of $U$. Then $\left\|\left.z \circ \Phi(\omega)\right|_{\mathscr{B}(\omega)}\right\| \leqq 1 / 2$ for all $\omega \in \Omega$. Hence, by Lemma 4.1,

$$
\begin{aligned}
& 1 \leqq \sup \left\{\left\|\left.z \circ \Phi(\omega)\right|_{\tilde{\mathfrak{z}}\left(\omega_{0}\right)}\right\|: \omega \in \Omega\right\} \\
& =\left\|\left.z \circ \Phi\right|_{\mathfrak{x}}\right\|=\left\|\left.z \circ \Phi\right|_{\mathscr{B}}\right\| \\
& =\sup \left\{\left\|\left.z \circ \Phi(\omega)\right|_{\mathscr{B}(\omega)}\right\|: \omega \in \Omega\right\} \leqq 1 / 2,
\end{aligned}
$$

a contradiction. Notice that, as $\Phi \in V_{\mathfrak{x}}$ is $\sigma$-weakly continuous, $\left\|\left.\Phi\right|_{\mathfrak{m}}\right\|=$ $\left\|\left.\Phi\right|_{\mathfrak{B}}\right\|$. Therefore, $\widetilde{\mathfrak{B}\left(\omega_{0}\right)}=\tilde{\mathfrak{B}}\left(\omega_{0}\right)$.

Conversely, assume $\overparen{\mathfrak{B}\left(\omega_{0}\right)}=\tilde{\mathfrak{B}}\left(\omega_{0}\right)$. Let $\delta$ be the conditional expectation to $\tilde{\mathfrak{B}}$ invariant by $\tau$. For each $a \in \mathfrak{A}$ and $\omega \in \Omega$,

$$
\begin{aligned}
\left\|\left.\varepsilon_{a}(\omega)\right|_{\mathfrak{P}(\omega)}\right\| & =\left\|\left.\varepsilon_{a}(\omega)\right|_{\mathfrak{\Re}(\omega)}\right\| \leqq\left\|\left.\varepsilon_{a}(\omega)\right|_{\mathfrak{\mathfrak { B }}(\omega)}\right\| \\
& =\left\|\left(\left.\varepsilon_{a}\right|_{\mathfrak{\mathfrak { F }}}\right)(\omega)\right\|=\left\|\left.\varepsilon_{\delta(a)}(\omega)\right|_{\mathfrak{\mathfrak { F }}(\omega)}\right\| \\
& =\varepsilon(|\delta(a)|)(\omega)
\end{aligned}
$$

by Lemma 4.3. In particular, since $\widetilde{\mathfrak{B}\left(\omega_{0}\right)}=\tilde{\mathfrak{B}}\left(\omega_{0}\right), \quad\left\|\left.\varepsilon_{a}\left(\omega_{0}\right)\right|_{\mathscr{B}\left(\omega_{0}\right)}\right\|=$ $\left\|\left.\varepsilon_{a}\left(\omega_{0}\right)\right|_{\mathfrak{F}\left(\omega_{0}\right)}\right\|=\varepsilon(|\delta(a)|)\left(\omega_{0}\right)$. Therefore, the function $\omega \rightarrow\left\|\left.\varepsilon_{a}(\omega)\right|_{\mathfrak{F}(\omega)}\right\|$ is upper semi-continuous at $\omega_{0}$. On the other hand, since $\left\|\left.\varepsilon_{a}(\omega)\right|_{\mathscr{P}(\omega)}\right\|=$ $\sup \left\{\left|\left\langle x(\omega), \varepsilon_{a}(\omega)\right\rangle\right| ; x(\omega) \in \mathfrak{B}(\omega), \| x(\omega)|| \leqq 1\right\}=\sup \left\{\left|\left\langle x(\omega), \varepsilon_{a}(\omega)\right\rangle\right| ; x(\omega) \in \mathfrak{B}(\omega)\right.$, $\|x(\omega)\|<1\}=\sup \left\{\left|\left\langle x(\omega), \varepsilon_{a}(\omega)\right\rangle\right| ; x \in \mathfrak{B},\|x\|<1\right\}$ for each $\omega \in \Omega$ and the function $\omega \rightarrow\left\langle x(\omega), \varepsilon_{a}(\omega)\right\rangle$ is continuous, the function $\omega \rightarrow\left\|\left.\varepsilon_{a}(\omega)\right|_{\mathscr{P}(\omega)}\right\|$ is lower semi-continuous on $\Omega$. Therefore, the function $\omega \rightarrow\left\|\left.\varepsilon_{a}(\omega)\right|_{\mathscr{F}(\omega)}\right\|$ is continuous at $\omega_{0}$. As $V_{\mathfrak{n}}$ is the uniform closure of the set $\left\{\varepsilon_{a} ; a \in \mathfrak{R}\right\}$, the function $\omega \rightarrow\left\|\left.\Phi(\omega)\right|_{g(\omega)}\right\|$ is continuous at $\omega_{0}$ for every $\Phi$ in $V_{\mathfrak{r}}$.

We remark that the above condition is not enough to assure $\mathfrak{B}=\tilde{\mathfrak{B}}$ as we see, for example, in the case of commutative algebras. In order to get weak closedness of $\mathfrak{B}$ we need the following condition.

THEOREM 4.9. Let $\mathfrak{B}$ be a $C^{*}$-subalgebra of $\mathfrak{A}$ containing $\mathscr{A}$, then the following conditions are equivalent;

(1) $\mathfrak{B}$ is a von Neumann subalgebra,

(2) For each $\varepsilon_{a} \in V_{\mathfrak{x}}$, there exists an element $v$ of $\mathfrak{B}$ with $\|v\| \leqq 1$

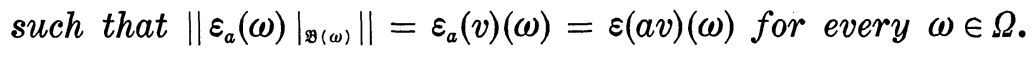


Proof. The implication $(1) \Rightarrow(2)$ has been already obtained from Lemma 1.1 and Theorem 4.4. We must show the converse. Since each element of $\tilde{\mathfrak{B}}$ is a linear combination of unitary elements, it suffices to show that each unitary element $u$ of $\tilde{\mathfrak{B}}$ belongs to $\mathfrak{B}$. From the assumption, there is an element $v \in \mathfrak{B}$ with $\|v\| \leqq 1$ such that

$$
\left\|\left.\varepsilon_{u}(\omega)\right|_{\Re(\omega)}\right\|=\varepsilon_{u}(v)(\omega)=\varepsilon(u v)(\omega)
$$

for every $\omega \in \Omega$. On the other hand, by Lemma 4.3 ,

$$
\| \varepsilon_{u}(\omega)|\tilde{\mathfrak{z}(\omega)}| \mid=\varepsilon(|u|)(\omega)=\varepsilon(1)(\omega)=1 .
$$

Since $\tilde{\mathfrak{B}}(\omega)=\widetilde{\mathfrak{B}(\omega)}$ by Theorem 4.8, this implies,

$$
\begin{aligned}
\left\|\left.\varepsilon_{u}(\omega)\right|_{\mathscr{P}(\omega)}\right\| & =\left\|\left.\varepsilon_{u}(\omega)\right|_{\Re(\omega)}\right\|=\left\|\left.\varepsilon_{u}(\omega)\right|_{\tilde{\mathfrak{F}}(\omega)}\right\| \\
& =1 .
\end{aligned}
$$

Hence, $\varepsilon(u v)=1$.

Let $u v=h^{+}-h^{-}+i k$ be the decomposition of $u v$ into positive and negative real parts and imaginary part. Then $\varepsilon(u v)=\varepsilon\left(h^{+}\right)-\varepsilon\left(h^{-}\right)+i \varepsilon(k)=1$, and $\varepsilon(k)=0$. Thus, $1=\varepsilon(u v) \leqq \varepsilon\left(h^{+}\right) \leqq 1$. Therefore, $\varepsilon\left(1-h^{+}\right)=0$ and $h^{+}=1$. Hence $\varepsilon\left(h^{-}\right)=0$ and $h^{-}=0$. Namely, $u v=1+i k$. However, the inequality

$$
1 \geqq\|u v\|=\|1+i k\|=\left\|1+k^{2}\right\|^{1 / 2}
$$

implies $k=0$. Hence $u v=1$, and $u=v^{*} \in \mathfrak{B}$. This completes the proof.

5. Restricted fields to closed subsets. Let $S$ be a closed subset of S. Then we can consider the $C^{*}$-algebra consisting of restrictions of all bounded weakly continuous fields with the norm

$$
\|\hat{x}\|_{S}=\sup \{\|x(\omega)\| ; \omega \in S\} .
$$

Denote this $C^{*}$-algebra by $\hat{\mathscr{A}} \mid S$. Let $\hat{V}_{\mathfrak{x}} \mid S$ be the space of all restrictions of those fields in $\hat{V}_{\mathfrak{a}}$ to $S$ with the norm

$$
\|\Phi\|_{S}=\sup \{\|\Phi(\omega)\| ; \omega \in S\} .
$$

Then one may expect that under certain conditions these two spaces of fields, $\hat{\mathscr{A}} \mid S$ and $\hat{V}_{x} \mid S$ inherit the original duality between $\hat{\mathscr{A}}$ and $\hat{V}_{x}$ in this localization. The answer to this question is nothing but recent Vesterstrøm's result ([16; Theorem 1.2]). In fact, as we have explained in $\S 2$, if we define the ideal $J=\left\{x ; \varepsilon\left(x^{*} x\right)(\omega)=0\right.$ for $\left.\omega \in S\right\}=\bigcap_{\omega \in S} \mathfrak{M}_{\omega}$ the quotient $C^{*}$-algebra $\mathfrak{X} / J=\mathfrak{A}(S)$ is isomorphic to $\hat{\mathfrak{A}} \mid S$ and $V_{\mathfrak{x}}(S)$ is isometric to $\hat{V}_{x} \mid S$. With these things, Vesterstrøm's theorem can be stated in the following way; 
TheOREM 5.1. The couple ( $\left(\hat{\mathscr{A}}\left|S, \hat{V}_{\mathfrak{Y}}\right| S\right)$ preserves the duality if $S$ is itself a countably decomposable hyperstonean space and $\hat{\mathfrak{A}} \mid S$ becomes $\sigma$ finite finite von Neumann algebra with the module predual $\hat{V}_{\mathbb{x}} \mid S$.

If one could prove an suitable extension theorem for bounded weakly continuous fields, one could give an another proof of the above theorem.

6. We have remarked before that in case of $\mathfrak{X}=\mathscr{A}=C(\Omega), C(\Omega)$ itself is the module predual space. Now, $C(\Omega)$ is a dense subset of $L^{1}(\Omega, \mu)$ and $L^{1}(\Omega, \mu)$ is considered as the space of all $\sigma$-weakly continuous linear functional on $C(\Omega)$. In connection with this, we give an adaptation of Theorem 1.3 in [16] in the following form. From this theorem one might be able to say that the space of all $\sigma$-weakly continuous $\mathscr{A}$-module mappings to $\mathscr{A}$ is the completion of $V_{\mathfrak{x}}$ in some sense.

TheoRem 6.1. Let $\Phi$ be a $\sigma$-weakly continuous $\mathscr{A}$-module mapping of $\mathscr{A}$ to $\mathscr{A}$, then there exist a sequence of orthogonal projections $\left\{z_{n}\right\}$ in $\mathscr{A}$ and a sequence of elements $\left\{\Phi_{n}\right\}$ in $V_{\mathfrak{x}}$ such that $\Phi=\sum_{n=1}^{\infty} z_{n} \circ \Phi_{n}$.

Proof. Since the functional $\tau \circ \Phi$ is $\sigma$-weakly continuous there exists a sequence $\left\{\Psi_{n}\right\}$ in $V_{\mathfrak{x}}$ such that $\tau \circ \Psi_{n}$ converges uniformly to $\tau \circ \Phi$. Henceforth we can employ the same arguments as in the proof of Theorem 1.3 in [16] and find a sequence $\left\{z_{n}\right\}$ of orthogonal projections in $\mathscr{A}$ and a sequence $\left\{\Phi_{n}\right\}$ of elements in $V_{\mathfrak{x}}$ with $\sum_{n=1}^{\infty} z_{n}=1$ such that

$$
\langle\Phi(x), \tau\rangle=\left\langle\sum_{n=1}^{\infty} \Phi_{n}\left(x z_{n}\right), \tau\right\rangle \text {. }
$$

Hence, for every $a \in \mathscr{A}$,

$$
\begin{aligned}
\langle a \Phi(x), \tau\rangle & =\langle\Phi(a x), \tau\rangle \\
& =\left\langle\sum_{n=1}^{\infty} \Phi_{n}\left(a x z_{n}\right), \tau\right\rangle=\left\langle a \sum_{n=1}^{\infty} \Phi_{n}\left(a z_{n}\right), \tau\right\rangle .
\end{aligned}
$$

Therefore, $\Phi(x)=\sum_{n=1}^{\infty} z_{n} \circ \Phi_{n}(x)$ for every $x \in \mathfrak{A}$ and $\Phi=\sum_{n=1}^{\infty} z_{n} \circ \Phi_{n}$.

So far as we are concerned with the case $\mathscr{A}=\not{3}$ the expectation $\varepsilon$ is unique as the $甘$-application. In general case we have

COROLLARY 6.2. Let $\varepsilon_{1}$ and $\varepsilon_{2}$ be conditional expectations to $\mathscr{A}$ and $\mathfrak{M}_{\omega}^{1}$ and $\mathfrak{M}_{\omega}^{2}$ be the ideals determined by $\varepsilon_{1}$ and $\varepsilon_{2}$ for $\omega \in \Omega$. Let $V_{\varkappa}^{1}$ and $V_{\mathfrak{\varkappa}}^{2}$ be the module predual of $\mathfrak{N}$ for $\varepsilon_{1}$ and $\varepsilon_{2}$. Then $\mathfrak{M}_{\omega}^{1}=\mathfrak{M}_{\omega}^{2}$ and $V_{\mathfrak{\varkappa}}^{1}(\omega)=$ $V_{\varkappa}^{2}(\omega)$ except for a non-dense subset of $\Omega$.

Proof. By the above theorem, there exists a non-dense subset $N$ in $\Omega$ such that $\varepsilon_{1}\left(\mathfrak{M}_{\omega}^{2}\right) \subset \mathfrak{M}_{\omega}^{2} \cap \mathscr{A}$ and $\varepsilon_{2}\left(\mathfrak{M}_{\omega}^{1}\right) \subset \mathfrak{M}_{\omega}^{1} \cap \mathscr{A}$ for every $\omega \in \Omega \sim N$. Then, if $x \in \mathfrak{M}_{\omega}^{2}, x^{*} x \in \mathfrak{M}_{\omega}^{2}$ and $\varepsilon_{1}\left(x^{*} x\right)(\omega)=0$. Hence $x \in \mathfrak{M}_{\omega}^{1}$. Similarly 
$x \in \mathfrak{M}_{\omega}^{1}$ implies $x \in \mathfrak{M}_{\omega}^{2}$. Thus $\mathfrak{M}_{\omega}^{1}=\mathfrak{M}_{\omega}^{2}$ for $\omega \in \Omega \sim N$. It follows from the unicity of the predual of the von Neumann algebra $\mathfrak{A}(\omega)$ that $V^{1}(\omega)=$ $V^{2}(\omega)$ for every $\omega \in \Omega \sim N$.

ADDED IN PROOF: (January 1973). During our revising the present paper, the article: S. Strătilă and L. Zsidó; “An algebraic reduction theory for $W^{*}$-algebras, I" has appeared. Following the idea based on Sakai [7] their arguments are concerned with the case of semi-finite von Neumann algebras. Thus they naturally lead to the same kind of context as ours, though our point of view and results presented here are somewhat different from their's.

\section{REFERENCES}

[1] J. Dixmier, Les algèbres d'operateurs dans l'espace hilbertien, Gauthier-Villars, Paris, 1957.

[2] J. Dixmier, Les $C^{*}$-algèbres et leurs représentations, Gauthier-Villars, Paris, 1964.

[ 3 ] J. Dixmier, Sur certains espaces considérés par M. H. Stone, Summa Brasil Math., 11 (1951), 151-182.

[4] J. Glimm, A Stone-Weierstrass theorem for $C^{*}$-algebras, Ann. of Math., 72 (1960), 216-244.

[5] H. Halpern, Module homomorphisms of a von Neumann algebra into its center, Trans. Amer. Math. Soc., 140 (1960), 183-193.

[6] K. H. HofmanN, Representations of algebras by continuous section, Bull. Amer. Math. Soc., 78-3 (1972), 291-373.

[7] S. SAKAI, The theory of $W^{*}$-algebras, Yale University, Lecture Note, 1962.

[8] S. SAKAI, A Radon-Nikodym theorem in $W^{*}$-algebra, Bull. Amer. Math. Soc., 73 (1965), 149-151.

[9] H. Tакемото, On the homomorphism of von Neumann algebra, Tôhoku Math. J., 21 (1969), 152-157.

]10] H. TAкемото, Complement to "On the homomorphism of von Neumann algebra", Tôhoku Math. J., 22 (1970), 210-211.

[11] M. TAKESAKI, The quotient algebra of a finite von Neumann algebra, Pacific Journ. Math., 36 (1971), 827-831.

[12] S. Teleman, Theory of harmonic algebras with applications to von Neumann algebras and cohomology of locally compact spaces, Lecture Notes in Mathematics 248 (1971), Springer-Verlag.

[13] J. Tomiyama, On the projection of norm one in $W^{*}$-algebras, Proc. Japan Acad., 33 (1957), 608-612.

[14] J. Tомічама, Topological representations of $C^{*}$-algebras, Tôhoku Math. J., 14 (1962), 187-204.

[15] H. UmEgakI, Conditional expectations in an operator algebra, Tôhoku Math. J., 6 (1954), 177-181.

[16] J. Vesterstrøm, Quotient of finite $W^{*}$-algebras, Bull. Amer. Math. Soc., 77 (1971), 235-238 and Journ. of Funct. Anal., 9-3 (1972), 322-335.

[17] J. Vesterstrøm, On the homomorphic image of the center of a $C^{*}$-algebra, Preprint series No. 18, 1970/71, Aarhus. 
[18] F. B. WRIGHT, A reduction theory for algebras of finite type, Ann. of Math., 60 (1954), 560-570.

Department of Mathematics

College of General Education

TôHOKU UNIVERSITY

SENDAI, JAPAN

AND

DEPARTMENT OF MATHEMATICS

YAMAGATA UNIVERSITY

YAMAGATA, JAPAN 
